\title{
Management of caesarean scar defects- a long neglected field-a short commentary
}

\section{Commentary}

In the recent decades incidence of caesarean sections (CS) has been increasing worldwide. In USA in 2007 percentage of CSs done was $>30 \%$, while rate in China increased to as high as $30-58 \%$ in 2010 and upto $80 \%$ in the private sector in Brazil. ${ }^{1,2}$ An estimated one to three quarters of CS delivery patients might develop a defect in their uterine scar. These CS scar defects can include uteroperitoneal fistula, niche, isthmocoele. ${ }^{3-5}$ Mostly they remain under-diagnosed and remain untreated at a very high proportion. The defect is a thinning of the myometrium at the site of hysterotomy/on the anterior uterine wall presenting as a breach at site of previous CS, which can be diagnosed by transvaginal sonography (TVS), saline infusion sonohysterography (SIS), hysteroscopy and magnetic resonance imaging (MRI) (Figure 1) (Figure 2). Women having had LSCS following labour have a distal defect more near the internal cervical os, while those having had an elective CS, the defect lies typically more proximal to the lower uterine segment. The wound healing might get affected in a woman who is a smoker, i.e. has diabetes mellitus (DM). These might lead to defect formation. The same also might be caused by surgical techniques like low hysterotomy (cervical), single layer or endometrial sparing closure, multiple CS deliveries or use of locking sutures (Figure 1) (Figure 2). ${ }^{5}$

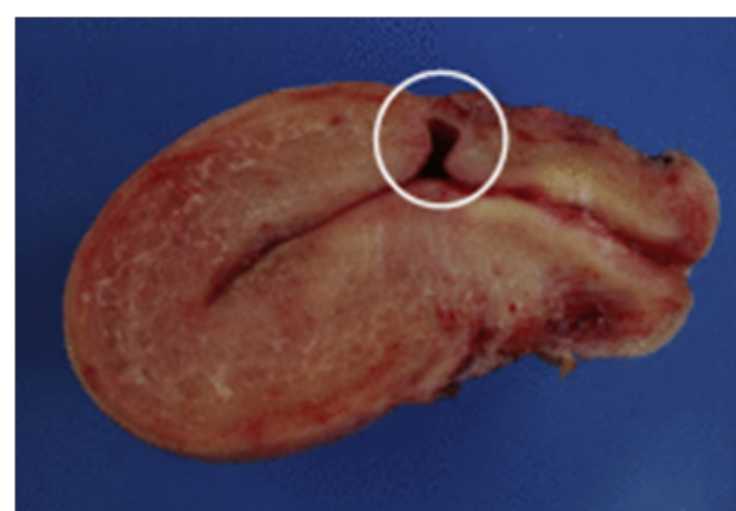

Figure I Courtesy ref no-6-Sagittal view of a frozen section from a hysterectomy specimen. A deep anterior defect covered with a thin layer of myometrium (white circle) can be observed at the level of the supposed site of CS.

Though isthmocele was $1^{\text {st }}$ described by Morris in 1995 and Jacobson $^{3}$ reported the first caesarean scar repair laparoscopically in 2003 the literature is still quiet scanty on this topic. With the rise in CS incidence worldwide CS scar defects and the symptoms associated along with risks include pain, bleeding, secondary infertility and ectopic scar pregnancies are also on an increase. The case reported by the group of Charles $\mathrm{R}$ was initially opened laparoscopically for adhesiolysis twice in lieu of secondary infertility, pelvic pain, menorrhagia but on worsening of the symptoms was passed on to colleagues who on dissection detected endometriosis on bladder. Further dissection of the uterus from anterior abdominal wall attachment and bladder they encountered a fistulous tract from the uterus to peritoneum which they named an uteroperitoneal fistula in
Volume 6 Issue 6 - 2018

\author{
Kulvinder Kochar Kaur,' Gautam Allahbadia, ${ }^{2}$ \\ Mandeep Singh ${ }^{3}$ \\ 'Scientific Director, Dr Kulvinder Kaur Centre for Human \\ Reproduction, India \\ ${ }^{2}$ Department of Obstetrics and Gynecology, Rotunda-A Centre \\ for Human reproduction, India \\ ${ }^{3}$ Department of Neurology, Swami Satyanand Hospital, India
}

Correspondence: Kulvinder Kochar Kaur, Scientific Director, Dr Kulvinder Kaur Centre for Human Reproduction, 721 G.T.B. Nagar, Jalandhar- I4400I, Punjab, India, Tel 9|-I8I-950I358|80, Email kulwinder.dr@gmail.com

Received: October 19,2018 | Published: November 19, 2018

2000.This was resection laparoscopically along with repair of the fistula and the defect was done. Subsequently the patient completely recovered symptomatically, conceiving spontaneously and delivered a live baby the next year.

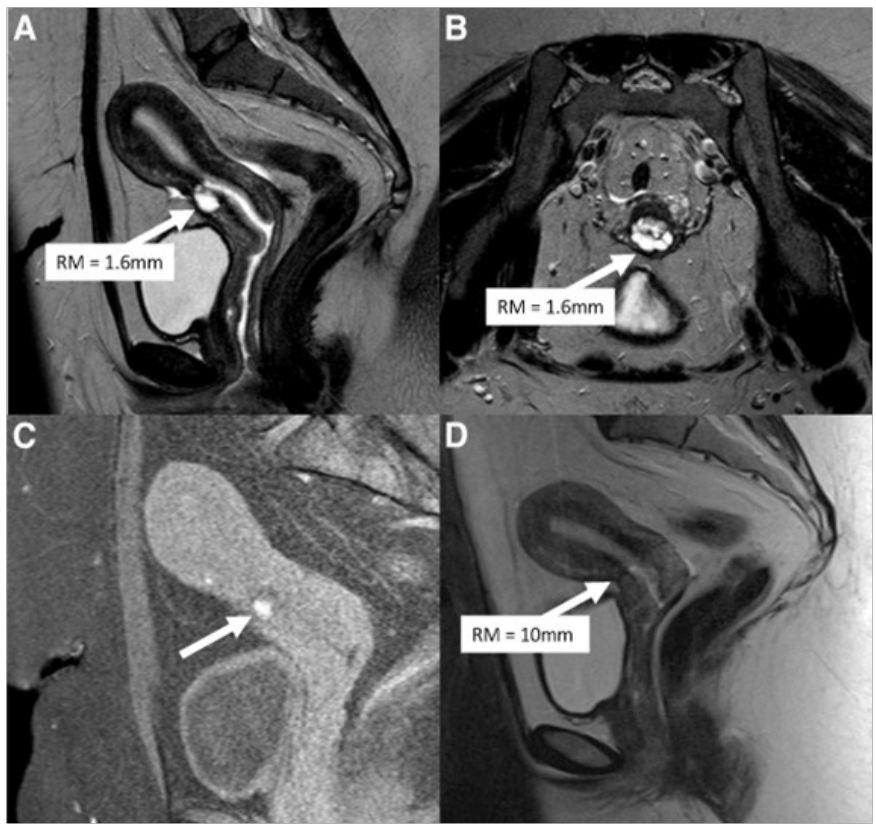

Figure 2 Courtesy ref 6-(A) Preoperative sagittal view of a T2-weighted image shows the residual myometrium (RM; white arrow) measuring $1.6 \mathrm{~mm}$, covering a deep cesarean scar defect. (B) Preoperative transversal view of a T2-weighted image from the same patient. The RM covering the scar (white arrow) is very thin. (C) Sagittal view of a TI-weighted image with saturation of fatty tissue showing hypersignals (white arrow) inside the cesarean scar defect, suggestive of blood retention. (D) Sagittal view of a T2-weighted image after laparoscopic repair. The defect is no longer visible, and the RM at the level of the isthmus (white arrow) measures $10 \mathrm{~mm}$. 
Recently, Donnez ${ }^{6}$ reported the largest prospective study till date. Patients with CS scar defects usually come with spotting following periods, secondary to accumulation of blood in the reservoir like niche. Pain, vaginal discharge, dysmenorrheal and dyspareunia is present in many. Secondary infertility is also common, possibly due to degradation of quality of sperm and cervical mucus by the accumulated blood. In case of conception by patients having CS defects risk of an ectopic CS pregnancy is very possible with the possibility of rupture. For diagnosis of niche one needs to examine the patient following menses when the endometrial lining is thin and blood has accumulated in the pouch at the CS delivery site. MRI or sonohysterogram can help in the diagnosis. A concave defect is often visualized on the anterior uterine wall on hysteroscopy. ${ }^{5}$ For surgical excision of this CS defect there are 3 methods, namely hysteroscopic resection, laparoscopic resection and repair with or without use of robotics or repair of the niche through a vaginal approach. Mostly laparoscopic resection of the CS defect is best for patients who desire fertility in view of better visualization and ability to re-approximate the myometrium with the use of 2 layer closures. Hysteroscopic resection might have role in selected cases .It is safe and efficient technique, useful for those not wanting pregnancy. As study was done by Raimondo in which they carried out hysteroscopic resection of a CS defect in 120 patients, most having postmenstrual bleeding and pain. Time taken was 8 'on average, with $80 \%$ of patients getting complete resolution of their symptoms. But a risk of bladder injury and uterine perforation exists if the myometrial thickness at the site of the defect is $<3 \mathrm{~mm} .{ }^{5}$ Thus an agreement existed between Nezhat and Donnez regarding repair of CS defects having $<3 \mathrm{~cm}$ thickness that a laporoscopic excision and repair be chosen. Donnez used very restrictive inclusion criteria to get a homogenous cohort. With the risk of rupture of thin niches having $<3$ mmof myometrium at the site of defect, ${ }^{7}$ Donnez included only patients meeting this criteria of having a thin myometrium $<3 \mathrm{~mm} .{ }^{6}$ As per the view of Nezhat even patients having myometrium measurements $>3 \mathrm{~mm}$ might also benefit from surgical treatment, where the surgeon can use either option of surgery be it laporoscopic repair or hysteroscopy. ${ }^{5}$ Also patients having bothering symptoms like postmenstrual bleeding, pain or infertility were included by Donnez although Nezhat group advocates use of this surgical repair irrespective of symptoms in those who require future fertility for reducing the risk of uterine rupture potentially. ${ }^{7}$

One good aspect of Donnez's study is the confirmation of histology following resection since it reconfirms the accuracy of MRI in confirming the defect and measuring the remaining myometrial thickness. In addition they found an additional 8/38(21\%) incidence of endometriosis in patients who had surgical excision of the CS defect. ${ }^{5}$ Coexistence of endometriosis with various female pelvic pathologies, like uterine fibroids corresponded with Nezhat group's experience having had faced endometriosis in their $1^{\text {st }}$ case as well. ${ }^{3}$ 6/8 patients who had pathologically proven endometriosis had experienced pain. Of these 2 had infertility alone without any pain or spotting .Thus a high suspicion for endometriosis needs to be kept at the time of laporoscopic surgery even if done for indications besides pain. Whether it is niche repair or any other surgery endometriosis that is visible needs excision. This thorough surgical treatment of endometriosis might help in preventing future pain, improve fertility and reduce the risk of endometriosis associated cancer. This article of Donnez again awakens the obstetricians and gynaecologists regarding the awareness of this defect and active measures be taken to relieve undiagnosed patients suffering from symptomatology for a long period.

Treatment can be determined by plan for future fertility. In those having niche thickness $>3 \mathrm{~mm}$ hysteroscopic approach is safe and may be better as $1^{\text {st }}$ line approach. Also symptomatic old patients who do not want fertility may be candidates for hysterectomy. Those having $<3 \mathrm{~mm}$ of myometrium at niche need laparoscopic resection with/ without robotics, and excise the fistulous tract and using multiple layer closure. ${ }^{8}$

\section{Reference}

1. Souza JP, Gulmezoglu A, Lumbiagnon P, et al. The WHO Global Survey on Maternal and Neonatal Research Group. Cesarean Section without Medical indications is associated with an increased risk of adverse short term maternal outcomes: the2004-2008 WHO Global Survey on Maternal and perinatal health. BMC Med. 2010;8:71.

2. Hamilton BE, Martin JA, Osterman MJ, et al. Births: final data for 2014. Natl Vital Stat Rep. 2015;64(12):1-64.

3. Jacobson MT, Osias J, Velasco A, et al. Laparoscopic repair of a utrpoeritoneal fistula. JSLS. 2003;7(4):367-369.

4. Raimondo G, Grifone G, Raimondo D, et al. Hysteroscopic treatment of symptomatic caesarean -induced isthmocoele:a prospective study. $J$ Minim Invasive Gynaecol. 2015;22(2):297-301.

5. Nezhat C, Soliemannjad R, Razavi GM, et al. Cesarean scar defect:what is it and how should it be treated? OBG Management. 2016;28(4):32-53.

6. Donnez O, Donnez J, Orellana R, et al. Gynecologic and obstetric outcomes after laparoscopic repair of a cesarean scar defect in a series of 38 women. Fertil Steril. 2017;107(1):289-296.

7. Bujold E, Jastrow N, Simoneau J, et al .Prediction of complete uterine rupture by sonographic evaluation of the lower uterine segment. $\mathrm{Am} \mathrm{J}$ Obstet Gynecol. 2009;201(3):320.e1-6.

8. Bhagavath B, Lindheim SR. Optimal management of symptomatic caesarean scar defects. Fertil Steril. 2018;110(3):417-418. 